\title{
Ist Forschung in der forensischen Psychiatrie ethisch zulässig?
}

\author{
Jakov Gather · Katrin Radenbach • Anna-Karina Jakovljević
}

(C) Springer-Verlag GmbH Deutschland, ein Teil von Springer Nature 2019

Die Frage, ob Forschung in der forensischen Psychiatrie ethisch zulässig ist, dürfte sich für viele als rhetorische Frage lesen. Warum sollte Forschung in einem Teilgebiet der Medizin nicht zulässig sein? Ist es nicht sogar ethisch geboten, für die wissenschaftlich begründete Entwicklung diagnostischer und therapeutischer Verfahren in der Psychiatrie Forschung durchzuführen, deren Ergebnisse Menschen mit psychischen Erkrankungen zu Gute kommen? Ist es nicht wichtiger, sich als psychiatrisch Forschende*r mit konkreten normativen Voraussetzungen für ethisch gerechtfertigte Forschung zu beschäftigen (Helmchen 2013), als die Zulässigkeit von Forschung grundsätzlich in Frage zu stellen?

Mit Blick auf die forensische Psychiatrie, einem Teilgebiet der Psychiatrie mit einem sehr spezifischen Aufgabenspektrum, erscheint es allerdings angebracht, sich auch mit der Frage nach der grundsätzlichen Zulässigkeit von Forschung zu beschäftigen. Dies gilt insbesondere für den klinisch-therapeutischen Teil der forensischen Psychiatrie, den sog. Maßregelvollzug. Die medizinisch-psychiatrische Behandlung

Dr. med. J. Gather, M.A. ( $₫)$

Institut für Medizinische Ethik und Geschichte der Medizin, Ruhr-Universität Bochum,

Malakowturm - Markstr. 258a, 44799 Bochum, Deutschland

E-Mail: jakov.gather@rub.de

Klinik für Psychiatrie, Psychotherapie und Präventivmedizin, LWL-Universitätsklinikum, Ruhr-Universität Bochum, Bochum, Deutschland

Dr. med. K. Radenbach

Klinik für Psychiatrie und Psychotherapie, Universitätsmedizin Göttingen,

Von-Siebold-Str. 5, 37075 Göttingen, Deutschland

E-Mail: katrin.radenbach@med.uni-goettingen.de

A.-K. Jakovljević, M.A.

Institut für Ethik und Geschichte der Medizin, Universitätsmedizin Göttingen,

Humboldtallee 36, 37073 Göttingen, Deutschland

E-Mail: ajakovl@gwdg.de 
von Straftäter*innen, die aufgrund einer psychischen Erkrankung zum Zeitpunkt der Tat nicht oder vermindert schuldfähig waren, gehört - neben der Begutachtung psychisch erkrankter Personen, die eine Straftat begangen haben - zu den wesentlichen Aufgaben der forensischen Psychiatrie. Die Behandlung findet in der Regel in speziell gesicherten Maßregelvollzugskliniken statt, in denen die Betroffenen auf Anordnung eines Gerichts zur „Besserung und Sicherung“ untergebracht sind. Allein im früheren Bundesgebiet inklusive Berlin waren dies im Jahr 2013 mehr als 10.000 Menschen (Statistisches Bundesamt 2015).

Die Besonderheiten der forensischen Psychiatrie und das spezifische Setting des Maßregelvollzugs gehen mit einer Vielzahl von ethischen Herausforderungen einher, die sich in anderen psychiatrischen Kontexten nicht oder zumindest nicht in einer so zugespitzten Form darstellen (Dudeck und Steger 2018). Dazu gehört auch die Frage nach den ethischen Voraussetzungen für Forschung mit im Maßregelvollzug untergebrachten Personen (Regehr et al. 2000; Munthe et al. 2010). Letztgenannte werden häufig als in doppelter Hinsicht vulnerabel und damit besonders schutzbedürftig angesehen, weil sie zum einen durch ihre psychische Erkrankung in ihrer Selbstbestimmungsfähigkeit eingeschränkt sein können (man denke nur an den großen Anteil an Menschen im Maßregelvollzug mit der Diagnose einer akuten oder chronischen Schizophrenie, einer Intelligenzminderung oder mit mehreren psychiatrischen Störungsbildern) und weil zum anderen - selbst bei gegebener Selbstbestimmungsfähigkeit - die Freiwilligkeit ihrer Entscheidung angesichts der unfreiwilligen Unterbringung in Frage steht (Arboleda-Flórez und Weisstub 2013; Birnbacher 2018).

Dass es sich bei der erhöhten Vulnerabilität und dem damit einhergehenden Risiko für einen Einschluss in Forschungsprojekte mit einem ungünstigen Nutzen-RisikoVerhältnis oder ohne gültigen Informed Consent nicht um ein rein hypothetisches Problem handelt, macht ein Blick in die jüngere Medizingeschichte deutlich. Diese weist bis weit in die zweite Hälfte des 20. Jahrhunderts hinein zahlreiche Beispiele für ethisch fragwürdige oder inakzeptable Forschung mit untergebrachten psychisch erkrankten Personen oder allgemein Menschen in Unfreiheit, vor allem Gefängnisinsass*innen, auf (Regehr et al. 2000; Elger 2009; Arboleda-Flórez und Weisstub 2013). Die internationalen Richtlinien des Council for International Organizations of Medical Sciences (CIOMS) fordern Forscher*innen und Ethikkommissionen daher auf, durch geeignete Schutzmechanismen die Rechte und das Wohl von vulnerablen Personen in psychiatrischen Einrichtungen oder Gefängnissen im Rahmen von Forschungsprojekten zu sichern (CIOMS 2016, S. 57-59).

Das deutsche Arzneimittelgesetz (AMG) geht an dieser Stelle einen strikten Weg und enthält unter den allgemeinen Voraussetzungen für klinische Prüfungen von Arzneimitteln ein Verbot für die Forschung mit im Maßregelvollzug untergebrachten Personen. Gemäß $§ 40$ Abs. 1 S. 3 Nr. 4 AMG dürfen klinische Prüfungen nur durchgeführt werden, wenn ,,die betroffene Person nicht auf gerichtliche oder behördliche Anordnung in einer Anstalt untergebracht ist". Dadurch werden in Deutschland zumindest bestimmte Studientypen im Maßregelvollzug verhindert, was dazu führt, dass es für manche, spezifisch im Maßregelvollzug angewendete medikamentöse Behandlungsmethoden kaum wissenschaftliche Evidenz gibt. Ein prominentes Beispiel hierfür ist die vor einigen Jahren trotz sorgfältiger Studienplanung nicht genehmig- 
te Studie zum Nutzen der testosteronsenkenden Behandlung von Sexualstraftätern mit dem Präparat Triptorelin (Briken et al. 2017). Doch wie verhält sich ein solches Verbot von Arzneimittelforschung im Maßregelvollzug zur - exemplarisch dem Niedersächsischen Maßregelvollzugsgesetz (Nds. MVollzG) entnommenen Forderung, dass untergebrachte Personen ,[...] Anspruch auf die nach dem aktuellen Stand des Wissens notwendige medizinische, therapeutische, pflegerische und pädagogische Behandlung und Untersuchung ihrer psychischen Krankheit, Störung oder Behinderung [...]“ ( $\$ 8$ Abs. 1 Nds. MVollzG) haben? Ist es ethisch gerechtfertigt, dass AMG-Studien mit untergebrachten Personen rechtlich nicht möglich sind, während Forschung außerhalb des AMG unter bestimmten Voraussetzungen durchgeführt werden kann, obwohl doch auch hier durch die Tatsache der unfreiwilligen Unterbringung die Freiwilligkeit der Zustimmung zur Studienteilnahme in Frage stehen dürfte? Welche Kriterien müssen erfüllt sein, damit eine Zustimmung zu einer Teilnahme an einem Forschungsprojekt als gültige, informierte Zustimmung gewertet wird, die ohne Einfluss von Zwang und Manipulation erfolgt ist? Über die Anzahl von Forschungsvorhaben im Bereich der forensischen Psychiatrie, die bislang von Ethikkommissionen aus ethischen oder rechtlichen Gründen abgelehnt worden sind, gibt es keine Daten. Und welche Rolle spielt eigentlich die Einschätzung der untergebrachten Personen selbst bei der Frage nach der ethischen Zulässigkeit von Forschung in der forensischen Psychiatrie? Mosbach et al. haben in einer Umfrage unter im Maßregelvollzug untergebrachten Personen herausgefunden, dass eine Mehrheit der Antwortenden Forschung im Maßregelvollzug für zulässig hält und sich ,frei fühlt“, einer Teilnahme an einem Forschungsprojekt zuzustimmen oder diese abzulehnen. Darüber hinaus zeigte die Mehrheit der Antwortenden eine grundsätzliche Bereitschaft, als Proband*in an potentiell eigennütziger Forschung teilzunehmen (Mosbach et al. 2015).

Vor dem dargelegten Hintergrund verfolgt dieses Themenheft das Ziel, die ethische Zulässigkeit von Forschung in der forensischen Psychiatrie aus einer interdisziplinären Perspektive zu diskutieren und eine weiterführende Debatte über dieses vergleichsweise selten in den Blick genommene forschungsethische Thema anzuregen. Diese weiterführende Debatte sollte nicht auf die forensische Psychiatrie oder auf bestimmte Studientypen beschränkt bleiben, denn vergleichbare ethische Fragen stellen sich beispielsweise auch bei Unterbringungen in der Allgemeinpsychiatrie oder bei qualitativ-empirischen Studien (Gather et al. 2019; Gieselmann et al. 2019; Scholten und Vollmann 2019).

Wir freuen uns, für dieses Themenheft ausgewiesene Wissenschaftler*innen aus verschiedenen Disziplinen als Autor*innen gewonnen zu haben. Ihre Beiträge knüpfen zum Teil an die Ergebnisse der interdisziplinären wissenschaftlichen Tagung zum Thema „Forschung mit besonders vulnerablen Gruppen in der Psychiatrie“ vom 7. Februar 2018 in Göttingen an. Organisiert wurde diese Tagung von der Arbeitsgruppe „Ethik in der Psychiatrie“ in der Akademie für Ethik in der Medizin e. V. sowie vom Institut für Ethik und Geschichte der Medizin der Universitätsmedizin Göttingen unter der Leitung von Claudia Wiesemann und dem Institut für Medizinische Ethik und Geschichte der Medizin der Ruhr-Universität Bochum unter der Leitung von Jochen Vollmann. 


\section{Die Beiträge im Einzelnen}

Im ersten Beitrag zeichnet Hanfried Helmchen die historische Entwicklung des Informed Consent in der psychiatrischen Forschung nach und hebt dabei unter anderem den zentralen Stellenwert einer sorgfältigen Konzeptualisierung und schließlich Prüfung der Einwilligungsfähigkeit bei Menschen mit psychischen Erkrankungen hervor. Darüber hinaus diskutiert er in seinem Artikel das vergleichsweise neue Instrument der Forschungsverfügung, bei dem er neben ungeklärten Fragen der Praktikabilität die Gefahr sieht, dass das Selbstbestimmungsrecht der Proband*innen nur noch mit Einschränkungen respektiert wird. Letzteres ist besonders hervorzuheben, da Gunnar Duttge und Melanie Steuer in ihrem Beitrag aus rechtswissenschaftlicher Perspektive die Frage untersuchen, ob und unter welchen Voraussetzungen Forschungsverfügungen eine „rechtliche Brücke“ für AMG-Studien im Maßregelvollzug sein können. Christian Prüter-Schwarte beschäftigt sich in seinem Artikel mit Versorgungs- und Prognoseforschung und damit mit Forschung außerhalb des AMG. Er entfaltet eine sozialethische Perspektive und plädiert vor diesem Hintergrund für eine Intensivierung der Forschungsbemühungen auf diesem Gebiet.

Ergänzt werden die Originalarbeiten durch eine Kontroverse zwischen den forensischen Psychiatern Jürgen L. Müller und Dirk Hesse, die sich in ihren Beiträgen sehr pointiert für bzw. gegen Forschung im Maßregelvollzug aussprechen. Ihre ethische Position entwickeln die beiden nicht zuletzt aus eigenen Erfahrungen mit Forschung im Maßregelvollzug, was die theoretische Diskussion um viele praxisrelevante Aspekte bereichert und die medizinethische Debatte an einigen Stellen herausfordern dürfte. Vor diesem Hintergrund verstehen wir die Kontroverse weniger als Abschluss dieses Themenhefts, sondern vielmehr als Ausgangspunkt und Anregung für weitere interdisziplinäre Überlegungen zu einer Forschungsethik in der forensischen Psychiatrie.

\footnotetext{
Danksagung Wir danken allen Autor*innen für ihre Beiträge und die vertrauensvolle Zusammenarbeit. Den externen Gutachter*innen danken wir für ihre hilfreichen Rückmeldungen zu den einzelnen Beiträgen. Wir danken der Schriftleitung von Ethik in der Medizin, dass dieses Themenheft ermöglicht wurde, und Thomas Schramme und Ralf J. Jox für die Unterstützung bei der Realisierung. Andrea Kähling danken wir für ihre gewissenhafte redaktionelle und organisatorische Hilfe.
}

J. Gather dankt außerdem dem Bundesministerium für Bildung und Forschung (BMBF) für die Förderung im Rahmen der BMBF-Forschungsgruppe „SALUS - Ethische Aspekte von Zwang in der Psychiatrie im Spannungsfeld von Selbstbestimmung, gesundheitlichem Wohl und Sicherheit“ (Förderkennzeichen: 01GP1792).

Interessenkonflikt J. Gather, K. Radenbach und A.-K. Jakovljević geben an, dass kein Interessenkonflikt besteht.

\section{Literatur}

Arboleda-Flórez J, Weisstub DN (2013) Forensic research with the mentally disordered offender. In: Kon$\operatorname{rad} \mathrm{N}$, Völlm B, Weisstub DN (Hrsg) Ethical issues in prison psychiatry. Springer, Dordrecht, S 103-122

Birnbacher D (2018) Selbstbestimmung und informed consent im Maßregelvollzug. In: Dudeck M, Steger F (Hrsg) Ethik in der Forensischen Psychiatrie und Psychotherapie. Medizinisch Wissenschaftliche Verlagsgesellschaft, Berlin, S 91-99 
Briken P, Müller JL, Berner W, Bödeker R-H, Vollmann J, Kasperk C, Koller M (2017) Vom Scheitern einer Studie in Maßregelvollzugskrankenhäusern. Klinische Prüfung zum additiven Effekt von Triptorelin auf die Wirksamkeit von Psychotherapie. Nervenarzt 88:480-485

Council for International Organizations of Medical Sciences (2016) International guidelines for healthrelated research involving humans. https://cioms.ch/wp-content/uploads/2017/01/WEB-CIOMSEthicalGuidelines.pdf. Zugegriffen: 15. Juli 2019

Dudeck M, Steger F (Hrsg) (2018) Ethik in der Forensischen Psychiatrie und Psychotherapie. Medizinisch Wissenschaftliche Verlagsgesellschaft, Berlin

Elger BS (2009) Ethische Prinzipien der Forschung mit Personen in Unfreiheit. Bioethica Forum 2:66-73

Gather J, Kalagi J, Otte I, Juckel G (2019) Interviewing a person with bipolar disorder under involuntary commitment: a case report. J Empir Res Human Res Ethics. https://doi.org/10.1177/ 1556264619847322

Gieselmann A, Efkemann SA, Scholten M (2019) Commentary 2: Qualitative research with vulnerable persons - how to ensure that burdens and benefits are proportional and fairly distributed. J Empir Res Human Res Ethics. https://doi.org/10.1177/1556264619847322

Helmchen H (Hrsg) (2013) Ethik psychiatrischer Forschung. Springer, Berlin, Heidelberg

Mosbach F, Hesse D, Müller JL, Wiesemann C (2015) Ausschluss von Patienten der Forensischen Psychiatrie von medizinischer Forschung - berechtigt aus Sicht der Betroffenen? In: Feuerstein G, Schramme T (Hrsg) Ethik der Psyche. Normative Fragen im Umgang mit psychischer Abweichung. Campus, Frankfurt am Main, S 505-516

Munthe C, Radovic S, Anckarsäter H (2010) Ethical issues in forensic psychiatric research on mentally disordered offenders. Bioethics 24:35-44

Regehr C, Edwardh M, Bradford J (2000) Research ethics and forensic patients. Can J Psychiatry 45:892-898

Scholten M, Vollmann J (2019) Commentary 1: research with persons under involuntary commitment-ethical issues surrounding competence and voluntariness. J Empir Res Human Res Ethics. https://doi.org/10.1177/1556264619847322

Statistisches Bundesamt (2015) Strafvollzugsstatistik. Im psychiatrischen Krankenhaus und in der Entziehungsanstalt aufgrund strafrichterlicher Anordnung Untergebrachte (Maßregelvollzug). https:// www.destatis.de/DE/Themen/Staat/Justiz-Rechtspflege/Publikationen/Downloads-StrafverfolgungStrafvollzug/krankenhaus-massregelvollzug-5243202149004.pdf?_blob=publicationFile. Zugegriffen: 15. Juli 2019 